August, 1992

\title{
Partition Functions for Heterotic WZW Conformal Field Theories
}

\author{
Terry Gannon \\ Mathematics Department, Carleton University \\ Ottawa, Ont., Canada K1S 5B6
}

Thus far in the search for, and classification of, 'physical' modular invariant partition functions $\sum N_{L R} \chi_{L} \chi_{R}^{*}$ the attention has been focused on the symmetric case where the holomorphic and anti-holomorphic sectors, and hence the characters $\chi_{L}$ and $\chi_{R}$, are associated with the same Kac-Moody algebras $\hat{g}_{L}=\hat{g}_{R}$ and levels $k_{L}=k_{R}$. In this paper we consider the more general possibility where $\left(\hat{g}_{L}, k_{L}\right)$ may not equal $\left(\hat{g}_{R}, k_{R}\right)$. We discuss which choices of algebras and levels may correspond to well-defined conformal field theories, we find the 'smallest' such heterotic (i.e. asymmetric) partition functions, and we give a method, generalizing the Roberts-Terao-Warner lattice method, for explicitly constructing many other modular invariants. We conclude the paper by proving that this new lattice method will succeed in generating all the heterotic partition functions, for all choices of algebras and levels. 


\section{Introduction}

Rational conformal field theories [1] consist of two nearly independent sectors, the holomorphic ("left-moving") and anti-holomorphic ("right-moving"), coupled via the partition function. The currents $J_{L}(z), J_{R}\left(z^{*}\right)$ corresponding to the two sectors of a WessZumino-Witten theory [2] generate two commuting Kac-Moody algebras $\hat{g}_{L}, \hat{g}_{R}$. Its partition function can be written as:

$$
Z\left(z_{L} z_{R} \mid \tau\right)=\sum N_{\hat{\lambda}_{L} \hat{\lambda}_{R}} \chi_{\hat{\lambda}_{L}}\left(z_{L}, \tau\right) \chi_{\hat{\lambda}_{R}}\left(z_{R}, \tau\right)^{*}
$$

where $\chi_{\hat{\lambda}_{L}}$ is the character of the representation of $\hat{g}_{L}$ with highest weight $\hat{\lambda}_{L}$ (similarly for $\chi_{\hat{\lambda}_{R}}$ ), the coefficients $N_{\hat{\lambda}_{L} \hat{\lambda}_{R}}$ are numbers (multiplicities), and the sum is over all highest weights with the levels $k_{L}, k_{R}$ fixed by the theory. All this will be described more carefully in the next section.

The algebras $\hat{g}_{L}, \hat{g}_{R}$ may or may not be isomorphic, and $k_{L}, k_{R}$ may or may not be equal. There are neither physical nor mathematical reasons why the case $\left(\hat{g}_{L}, k_{L}\right) \neq$ $\left(\hat{g}_{R}, k_{R}\right)$ should be avoided, and in fact experience from string theory [3] hints that this case could include some interesting theories. Any such asymmetric theory, and its partition function, shall be called heterotic.

There are three properties the sum in eq.(1.1) must satisfy in order to be the partition function of a physical conformal field theory:

(P1) it must be modular invariant. This is equivalent to the two conditions:

$$
\begin{gathered}
Z\left(z_{L} z_{R} \mid \tau+1\right)=Z\left(z_{L} z_{R} \mid \tau\right) \\
\exp \left[-\pi i\left(k_{L} z_{L}^{2} / \tau-k_{R} z_{R}^{* 2} / \tau^{*}\right)\right] Z\left(z_{L} / \tau, z_{R} / \tau \mid-1 / \tau\right)=Z\left(z_{L} z_{R} \mid \tau\right)
\end{gathered}
$$

(P2) the coefficients $N_{\lambda_{L} \lambda_{R}}$ in eq.(1.1) must be non-negative integers; and

(P3) uniqueness of the vacuum: $N_{\hat{\lambda}_{L} \hat{\lambda}_{R}}=1$ for $\hat{\lambda}_{L}=k_{L} \hat{\beta}_{0 L}, \hat{\lambda}_{R}=k_{R} \hat{\beta}_{0 R}$ (these are the highest weights of the singlet representations of levels $k_{L}, k_{R}$, respectively; $\hat{\beta}_{0 L}$ and $\hat{\beta}_{0 R}$ are the fundamental weights (see [10]) associated with the 0-nodes of the Dynkin diagrams of $\hat{g}_{L}$ and $\hat{g}_{R}$, respectively). In the following sections we switch notation from affine weights $\hat{\lambda}$ to horizontal weights $\lambda$, and this condition reduces to $N_{00}=1$. If the function $Z$ in (1.1) satisfies (P1), we will call it an invariant; if in addition it satisfies (P2) and (P3) we will call it a physical invariant. These properties are necessary for $Z$ to be the partition function of a sensible theory, but they are not sufficient. In this paper we will be interested in the construction and classification of all physical invariants corresponding to a given choice of algebras and levels, but we will not address the question of which of these actually correspond to well-defined conformal field theories.

Much work has been done recently on finding and classifying physical invariants (see e.g. [4]). However, so far the attention of researchers has rested almost exclusively on the special case where $\hat{g}_{L}=\hat{g}_{R}=\hat{g}$ and $k_{L}=k_{R}=k$ (perhaps the only notable exception is level 1, the canonical example being the heterotic string). Such an invariant will be called symmetric. In the following section one such approach [5], due to Warner and, independently, Roberts and Terao, shall be described. 
Perhaps the principal reason for the general absence of work on heterotic invariants is that the standard tools developed for the symmetric case (e.g. using simple currents [6], or automorphisms of the fusion rules of extended algebras [7]) are not as easy to apply in the heterotic case. Those algebraic techniques provide a very elegant derivation of many symmetric physical invariants, particularly those lying on infinite series, but some of the so-called exceptional physical invariants are less tractible from that perspective. Many exceptional invariants can be constructed using conformal embeddings [8], but not all can. A major strength of the Roberts-Terao-Warner lattice approach is that all invariants are placed on an equal footing. Moreover, it is a complete method [9], and for small ranks and levels is very practical. These qualities make it particularly suited to the heterotic case, where we will find that there are only exceptional physical invariants.

Their lattice method extends naturally to the heterotic case. However, the resulting method will fail to find any nonzero modular invariants, unless the algebras and levels involved satisfy excessively strong conditions. Fortunately, it is possible to generalize their method, in two independent ways, so that the completeness of the symmetric case is transferred into a completeness for the heterotic case: this generalized lattice method will find all possible heterotic partition functions (see Thm.E).

This paper is concerned with the search for heterotic physical invariants. In Sec.2 we review the lattice approach of [5] for constructing symmetric invariants. We generalize it in Sec.3. In Sec.4 we analyse this new method. We find a necessary condition on the algebras and levels required for the existence of physical invariants. In Sec.5 we give explicit examples, and use the analysis of Sec.4 to find all heterotic physical invariants of smallest 'total rank' (see eqs.(5.3)). In the final section we provide a rigorous proof of the completeness of this new method.

\section{The lattice approach of Roberts-Terao-Warner}

In this section we will restrict our attention to the symmetric case where $\hat{g}_{L}=\hat{g}_{R}=$ $\hat{g}$ and $k_{L}=k_{R}=k$. We will review the method of Roberts-Terao-Warner, changing their notation and presentation somewhat. But first we will briefly introduce some of the notation involved with Kac-Moody algebras (see [10,11] for details).

Let $g$ be a (simple) finite-dimensional Lie algebra of rank $n$, and let $\alpha_{1}^{\vee}, \ldots, \alpha_{n}^{\vee}$ be its simple coroots. The span (over the integers $\mathbf{Z}$ ) of these coroots is called the coroot lattice of $g$ and will be denoted by $M=M_{g}$. These lattices $M$ are listed in the appendix for each choice of $g$. There exist vectors $\beta_{1}, \ldots, \beta_{n}$ in the dual $M^{*}$ of the lattice $M$ satisfying $\beta_{i} \cdot \alpha_{j}^{\vee}=\delta_{i j}$; these vectors span $M^{*}$ and are called the fundamental weights of $g$. Let $\rho=\sum_{i} \beta_{i}$

Each $g$ also has a set of colabels $a_{i}^{\vee}, i=1, \ldots, n$. The number $1+\sum a_{i}^{\vee}$ is called the dual Coxeter number and is denoted by $h^{\vee}$. For each $k=0,1,2, \ldots$ define

$$
P_{+}(g, k) \stackrel{\text { def }}{=}\left\{\sum_{i=1}^{n} m_{i} \beta_{i} \mid m_{i} \in \mathbf{Z}, \quad 0 \leq m_{i}, \sum_{i=1}^{n} m_{i} a_{i}^{\vee} \leq k\right\} \subset M^{*} .
$$

Now consider the untwisted affine extension $\hat{g}=g^{(1)}$ of $g$. It turns out that the properties of $\hat{g}$ can be expressed in terms of those of $g$. The details will not be given 
here. Any integrable irreducible representation of $\hat{g}$ is associated with a positive number $k$, called its level, and a (horizontal) highest weight vector $\lambda \in P_{+}(g, k)$. For example, the singlet representation considered in (P3) corresponds to $\lambda=0$. A representation has the (normalized) character denoted by $\chi_{\lambda}^{g, k}(u, z, \tau)$. Here, $u$ and $\tau$ are complex numbers, $\operatorname{Im}(\tau)>0$, and $z$ is a complex vector lying in

$$
\mathbf{C} \otimes M \stackrel{\text { def }}{=}\left\{\sum_{i} c_{i} x_{i} \mid c_{i} \in \mathbf{C}, \quad x_{i} \in M\right\} .
$$

(We will use that notation throughout this paper; $\mathbf{R} \otimes \Lambda$ and $\mathbf{Q} \otimes \Lambda$ are defined similarly, for any lattice $\Lambda$.) The variable $u$ is not relevant for what follows and will be ignored (i.e. set equal to 0). Most writers consider the partition functions (1.1) to involve the restricted characters $\chi_{\lambda}^{g, k}(\tau)$ where $z$ is also set equal to 0. (However in [12] it is argued that for conformal field theories with $c \geq 1$ - which is the case of interest here - these restricted partition functions cannot carry enough information to specify the theory and so $z$ should be retained.) In this paper we will retain the vectors $z$. Of course the restricted partition functions can be recovered at the end by substituting in $z=0$.

We will only consider the case where the algebras $\hat{g}_{L}, \hat{g}_{R}$ are untwisted affine algebras. The approach of Roberts-Terao-Warner (henceforth RTW) starts from the Weyl-Kac character formula for these algebras. But before we can state it, we need the following definitions.

Given any positive definite lattice $\Lambda$ and any vector $v \in \mathbf{Q} \otimes \Lambda$, the translate $v+\Lambda$ is called a glue class. The theta series of that glue class is defined to be

$$
\Theta(v+\Lambda)(z \mid \tau) \stackrel{\text { def }}{=} \sum_{x \in v+\Lambda} \exp \left[\pi i \tau x^{2}+2 \pi i z \cdot x\right] .
$$

Here $\tau \in \mathbf{C}$, and $z$ is a complex vector lying in $\mathbf{C} \otimes \Lambda$. Since $\Lambda$ is positive definite, this converges and in fact is analytic for all such $z$ and any $\tau$ in the upper half plane. Given any lattice $\Lambda$ and positive number $\ell$, we write $\Lambda^{(\ell)}$ for the (positive definite) scaled lattice $\sqrt{\ell} \Lambda$, and $\Lambda^{(-\ell)}$ for the corresponding negative definite scaled lattice.

An integral lattice is one in which all dot products are integers. An even lattice is both integral, and has only even norms. An odd lattice is an integral lattice with at least one odd normed vector. A self-dual lattice $\Lambda$ is one which equals its dual $\Lambda^{*}$. Finally, by a gluing $\Lambda$ of $\Lambda_{0}$, we mean that $\Lambda_{0}$ is a sublattice in $\Lambda$ of finite index. Thus a gluing of $\Lambda_{0}$ is precisely any lattice which can be written as a finite disjoint union of glue classes of $\Lambda_{0}$.

For a discussion of glue classes and their uses, see for example [13]. The theta series of lattices are addressed for example in [14].

Now we return to the context of the algebra $g . M$ is its coroot lattice and $W(g)$ is its Weyl group. Given a transformation $w \in W(g)$, we can define its $\operatorname{sign} \epsilon(w)=\operatorname{det}(w) \in$ $\{ \pm 1\}$.

The Weyl-Kac character formula can now be written as:

$$
\begin{aligned}
\chi_{\lambda}^{g, k}(z, \tau) & =\frac{\sum_{w \in W(g)} \epsilon(w) \Theta\left(\frac{\lambda+\rho}{\sqrt{k+h^{\vee}}}+M^{\left(k+h^{\vee}\right.}\right)\left(\sqrt{k+h^{\vee}} w(z) \mid \tau\right)}{D_{g}(z \mid \tau)}, \\
D_{g}(z \mid \tau) & \stackrel{\text { def }}{=} \sum_{w \in W(g)} \epsilon(w) \Theta\left(\frac{\rho}{\sqrt{h^{\vee}}}+M^{\left(h^{\vee}\right)}\right)\left(\sqrt{h^{\vee}} w(z) \mid \tau\right) .
\end{aligned}
$$


Note that eq. (2.2a) allows us to define $\chi_{\lambda}^{g, k}$ for any $\lambda \in M^{*}$. However, it can be shown (see [10]) that for any $\lambda \in M^{*}$ and $k \geq 0$, either

$$
\chi_{\lambda}^{g, k}(z, \tau)=0
$$

for all $z$ and $\tau$, or there exists a unique $\lambda^{\prime} \in P_{+}(g, k)$ and $\epsilon \in\{ \pm 1\}$ such that

$$
\chi_{\lambda}^{g, k}(z, \tau)=\epsilon \chi_{\lambda^{\prime}}^{g, k}(z, \tau)
$$

for all $z$ and $\tau$.

The central idea of the RTW lattice method is as follows.

By $\left(M^{(\ell)} ; M^{(\ell)}\right)$ we mean the $2 n$-dimensional indefinite lattice $M^{(\ell)} \oplus M^{(-\ell)}$; we will denote its vectors by $\left(x_{L} ; x_{R}\right)$ in the obvious way. We are interested here in the choice $\ell=k+h^{\vee}$. Let $\Lambda$ be any even self-dual gluing of $\Lambda^{g, k} \stackrel{\text { def }}{=}\left(M^{\left(k+h^{\vee}\right)} ; M^{\left(k+h^{\vee}\right)}\right)$. That implies that

$$
\Lambda^{g, k} \subset \Lambda=\Lambda^{*} \subset \Lambda^{g, k *} .
$$

We will also write the vectors of $\Lambda$ in the form $\left(x_{L} ; x_{R}\right)$.

Define the function

$$
\begin{aligned}
W Z_{\Lambda}(g, k)\left(z_{L} z_{R} \mid \tau\right) & \stackrel{\text { def }}{=} \sum_{w_{L}, w_{R} \in W(g)} \epsilon\left(w_{L}\right) \epsilon\left(w_{R}\right) \sum_{\left(x_{L} ; x_{R}\right) \in \Lambda} \exp \left[\pi i \tau x_{L}^{2}-\pi i \tau^{*} x_{R}^{2}\right] \cdot \quad \\
& \cdot \exp \left[2 \pi \sqrt{k+h^{\vee}} i\left(w_{L}\left(z_{L}\right) \cdot x_{L}-w_{R}\left(z_{R}\right)^{*} \cdot x_{R}\right)\right] / D_{g}\left(z_{L} \mid \tau\right) D_{g}\left(z_{R} \mid \tau\right)^{*}
\end{aligned}
$$

Because $\Lambda$ is a gluing of $\Lambda^{g, k}$, eq. $(2.2 a)$ tells us we can write $W Z_{\Lambda}(g, k)\left(z_{L} z_{R} \mid \tau\right)$ as a sum of terms looking like

$$
\chi_{\lambda_{L}}^{g, k}\left(z_{L}, \tau\right) \cdot \chi_{\lambda_{R}}^{g, k}\left(z_{R}, \tau\right)^{*}
$$

for $\lambda_{L}, \lambda_{R} \in M^{*}$. Eqs.(2.3) now tell us that $W Z_{\Lambda}(g, k)\left(z_{L} z_{R} \mid \tau\right)$ can be written as a linear combination (over $\mathbf{Z}$ ) of terms like that in eq.(2.5), with $\lambda_{L}, \lambda_{R}$ now lying in $P_{+}(g, k)$. Also, because $\Lambda$ is even, $(1.2 a)$ is satisfied, and because $\Lambda$ is self-dual, it can be shown (using Poisson's equation or more directly eq.(3.10b) in [14], together with Lemma 13.8 in [10]) that $(1.2 b)$ holds.

The RTW method suggests we look at these $W Z_{\Lambda}(g, k)$. We know from the previous paragraph that these functions are in the form of eq.(1.1), and that property (P1) is satisfied. In general, (P2) and (P3) will not be. However, any linear combination

$$
\sum_{i} \ell_{i} W Z_{\Lambda_{i}}(g, k)\left(z_{L} z_{R} \mid \tau\right)
$$

which satisfies (P2) and (P3), will be a physical invariant when each $\Lambda_{i}$ is an even self-dual gluing of $\Lambda^{g, k}$.

Roberts and Terao [5] expressed all known physical invariants corresponding to $g=A_{1}$ or $g=A_{2}$ in the form (2.6). This author has shown in [9] that all (symmetric) physical invariants associated to any $g$, of any level $k$, must necessarily be expressible in this form. 


\section{Generalizing the RTW method}

The extension of eq.(1.1) to semi-simple algebras is trivial. By a type $\mathcal{T}$ we mean the collection

$$
\begin{aligned}
\mathcal{T} & =\left(\mathcal{T}_{L} ; \mathcal{T}_{R}\right), \text { where } \\
\mathcal{T}_{L} & =\left(\left\{g_{L 1}, k_{L 1}\right\},\left\{g_{L 2}, k_{L 2}\right\}, \ldots,\left\{g_{L l}, k_{L l}\right\}\right), \\
\mathcal{T}_{R} & =\left(\left\{g_{R 1}, k_{R 1}\right\}, \ldots,\left\{g_{R r}, k_{R r}\right\}\right) .
\end{aligned}
$$

Here each $g_{L i}$ and $g_{R j}$ is a simple finite-dimensional Lie algebra, and each $k_{L i}$ and $k_{R j}$ is a nonnegative integer. For shorthand we will write

$$
\begin{aligned}
\Lambda\left(\mathcal{T}_{L}\right) & =M_{g_{L 1}}^{\left(k_{L 1}+h_{L 1}^{\vee}\right)} \oplus \cdots \oplus M_{g_{L l}}^{\left(k_{L l}+h_{L l}^{\vee}\right)}, \\
P_{+}\left(\mathcal{T}_{L}\right) & =P_{+}\left(g_{L 1}, k_{L 1}\right) \times \cdots \times P_{+}\left(g_{L l}, k_{L l}\right), \\
\chi_{\lambda_{L}}\left(\mathcal{T}_{L}\right)\left(z_{L}, \tau\right) & =\chi_{\lambda_{L 1}}^{g_{L 1}, k_{L 1}}\left(z_{L 1}, \tau\right) \cdots \chi_{\lambda_{L l}}^{g_{L l}, k_{L l}}\left(z_{L l}, \tau\right),
\end{aligned}
$$

with similar definitions for $\mathcal{T}_{R}$, where ' $\oplus$ ' in $(3.2 a)$ denotes the orthogonal direct sum of lattices and ' $X$ ' in $(3.2 b)$ denotes the cartesian product of sets, and where $\lambda_{L}=$ $\left(\lambda_{L 1}, \ldots, \lambda_{L l}\right) \in \Lambda\left(\mathcal{T}_{L}\right)^{*}$ and $z_{L}=\left(z_{L 1}, \ldots, z_{L l}\right) \in \mathbf{C} \otimes \Lambda\left(\mathcal{T}_{L}\right)$.

Then (1.1) becomes

$$
Z(\mathcal{T})\left(z_{L} z_{R} \mid \tau\right)=\sum_{\lambda_{L}, \lambda_{R}} N_{\lambda_{L} \lambda_{R}} \chi_{\lambda_{L}}\left(\mathcal{T}_{L}\right)\left(z_{L}, \tau\right) \chi_{\lambda_{R}}\left(\mathcal{T}_{R}\right)\left(z_{R}, \tau\right)^{*},
$$

where the sum is over all $\lambda_{L} \in P_{+}\left(\mathcal{T}_{L}\right), \lambda_{R} \in P_{+}\left(\mathcal{T}_{R}\right)$.

Because for any $g$,

$$
P_{+}(g, 0)=\{0\} \text { and } \chi_{0}^{g, 0}(z, \tau)=1,
$$

we can and usually will assume in (3.1) that no levels $k_{L i}, k_{R j}$ equal 0 . When all $l+r$ levels are positive, $\mathcal{T}$ is said to be a positive type. By definition, we will insist that the type of any (nonconstant) invariant be positive. We will call an invariant $Z$, or its type $\mathcal{T}$, symmetric if $l=r$ and, up to a rearrangement of the indices, $\left(g_{L i}, k_{L i}\right)=\left(g_{R i}, k_{R i}\right)$. If not symmetric, they will be called heterotic.

Let $n_{L i}, n_{R j}$ be the ranks of $g_{L i}, g_{R j}$, and define $n_{L}=n_{L 1}+\cdots+n_{L l}, n_{R}=$ $n_{R 1}+\cdots+n_{R r}$. By the total rank of an invariant (or its type) we mean the number $n_{L}+n_{R}=n_{L 1}+\cdots+n_{R r}$.

In this section we are concerned with the search for physical invariants of arbitrary type. The idea is to generalize the RTW approach, i.e. to find a class of lattices $\Lambda$ and some function $f_{\Lambda}\left(z_{L} z_{R} \mid \tau\right)$ with the property that

$$
\begin{aligned}
W f_{\Lambda}\left(z_{L} z_{R} \mid \tau\right) \stackrel{\text { def }}{=} & \sum_{w_{L i}, w_{R j}} \epsilon\left(w_{L 1}\right) \cdots \epsilon\left(w_{R r}\right) . \\
& \cdot \frac{f_{\Lambda}\left(\sqrt{k_{L 1}+h_{L 1}^{\vee}} w_{L 1}\left(z_{L 1}\right), \ldots, \sqrt{k_{R r}+h_{R r}^{\vee}} w_{R 1}\left(z_{R 1}\right) \mid \tau\right)}{D_{g_{L 1}}\left(z_{L 1} \mid \tau\right) \cdots D_{g_{R r}}\left(z_{R r} \mid \tau\right)^{*}}
\end{aligned}
$$


(the sum is over all $w_{L i} \in W\left(g_{L i}\right), w_{R j} \in W\left(g_{R j}\right)$ ) can firstly (i) be written as a linear combination of terms of the form

$$
\chi_{\lambda_{L}}\left(\mathcal{T}_{L}\right)\left(z_{L}, \tau\right) \cdot \chi_{\lambda_{R}}\left(\mathcal{T}_{R}\right)\left(z_{R}, \tau\right)^{*},
$$

for $\lambda_{L} \in P_{+}\left(\mathcal{T}_{L}\right), \lambda_{R} \in P_{+}\left(\mathcal{T}_{R}\right)$, and secondly (ii) that for all $\Lambda$ in that class of lattices the function $W f_{\Lambda}$ has the correct modular behaviour (see eqs.(1.2)).

The obvious extension of the RTW lattice method to this more general situation is to let the lattices $\Lambda$ in (3.5) be even self-dual gluings of the indefinite lattice

$$
\Lambda(\mathcal{T}) \stackrel{\text { def }}{=}\left(\Lambda\left(\mathcal{T}_{L}\right) ; \Lambda\left(\mathcal{T}_{R}\right)\right),
$$

and to define the function $W Z_{\Lambda}(\mathcal{T})\left(z_{L} z_{R} \mid \tau\right)$ as the obvious analogue of (2.4), by choosing $f_{\Lambda}=\Theta(\Lambda)$. However, by a well-known theorem (see e.g. [13]) such $\Lambda$ could exist only when the ranks $n_{L i}, n_{R j}$ (which equal the dimensions of the coroot lattices $M_{g_{L i}}, M_{g_{R j}}$ ) satisfy the congruence $n_{L} \equiv n_{R}(\bmod 8)$. This excessively strong condition suggests this extension is not sufficiently general.

There are two directions in which we will generalize the RTW method. We will consider a more general $f_{\Lambda}$, which will allow us to use odd self-dual lattices as well as even ones. Secondly, we will use (3.4) to allow us to use other types $\mathcal{T}^{\prime}$ in our search for invariants of type $\mathcal{T}$.

Before we continue we should explicitly write down the modular behaviour of the denominator $D_{g}$ of the Weyl-Kac formula, eq.(2.2).

$$
\begin{aligned}
D_{g}(z \mid \tau+1) & =\exp \left[\pi i \rho^{2} / h^{\vee}\right] D_{g}(z \mid \tau) \\
D_{g}(z / \tau \mid-1 / \tau) & =\left(\frac{\tau}{i}\right)^{n / 2} \exp \left[\pi i h^{\vee} z^{2} / \tau\right] \exp \left[3 \pi i\left\|\Delta_{+}\right\| / 2\right] D_{g}(z \mid \tau)
\end{aligned}
$$

where $\rho$ is defined in the previous section, and where $\left\|\Delta_{+}\right\|$denotes the number of positive roots of $g$. The numbers $\rho^{2}$ and $\left\|\Delta_{+}\right\|$for each $g$ are included in the appendix. The proof of (3.7a) follows immediately from the fact that the coroot lattice $M$ is even for any Lie algebra $g$. The proof of (3.7b) is given in Lemma 13.8 of [10].

Let $\Lambda$ be any lattice (not necessarily self-dual), and let $u=\left(u_{L} ; u_{R}\right), v=\left(v_{L} ; v_{R}\right) \in$ $\mathbf{R} \otimes \Lambda(\mathbf{R} \otimes \Lambda$ is defined in the fourth paragraph of Sec.2). A fairly general lattice function which is reasonably well-behaved under $\tau \rightarrow-1 / \tau$ is the following function (similar to one found in [15] in the context of string theory, and related to the theta functions of rational characteristic in [16]):

$$
\begin{aligned}
A^{u, v}(\Lambda)\left(z_{L} z_{R} \mid \tau\right)= & \sum_{\left(x_{L} ; x_{R}\right) \in \Lambda} \exp \left[\pi i \tau\left(x_{L}+u_{L}\right)^{2}-\pi i \tau^{*}\left(x_{R}+u_{R}\right)^{2}\right] . \\
& \cdot \exp \left[2 \pi i\left\{\left(z_{L}+v_{L}\right) \cdot\left(x_{L}+u_{L}\right)-\left(z_{R}^{*}+v_{R}\right) \cdot\left(x_{R}+u_{R}\right)\right\}\right] .
\end{aligned}
$$

Then Poisson's eq. gives us (for integral $\Lambda$ )

$$
\begin{aligned}
A^{u, v}(\Lambda)\left(z_{L} / \tau, z_{R} / \tau \mid-1 / \tau\right)= & \frac{\left(\frac{\tau}{i}\right)^{n_{L} / 2}\left(\frac{-\tau^{*}}{i}\right)^{n_{R} / 2}}{\sqrt{|\Lambda|}} \exp \left[\pi i z_{L}^{2} / \tau-\pi i z_{R}^{* 2} / \tau^{*}\right] . \\
& \cdot e^{2 \pi i u \cdot v} \sum_{[g] \in \Lambda^{*} / \Lambda} A^{v+g,-u}(\Lambda)\left(z_{L} z_{R} \mid \tau\right) .
\end{aligned}
$$


We would like to make the choice $f_{\Lambda}=A^{u, v}(\Lambda)$ in (3.5), which suggests that we demand $\Lambda^{*}=\Lambda, v \equiv u(\bmod \Lambda)$, and $-u \equiv v\left(\bmod \Lambda^{*}\right)$, i.e. that $\Lambda$ be self-dual, $u=v$, and $2 u \in \Lambda$.

Eqs.(1.2a) and (3.7a) suggest that $A^{u, v}(\Lambda)\left(z_{L} z_{R} \mid \tau+1\right)$ should equal $A^{u, v}(\Lambda)\left(z_{L} z_{R} \mid \tau\right)$ up to some constant factor, which in turn suggests that we should require $(x+u)^{2}$ be independent $(\bmod 2)$ of $x \in \Lambda$. This holds iff

$$
x^{2}+2 x \cdot u \equiv 0(\bmod 2) \forall x \in \Lambda \text {. }
$$

Given any integral $\Lambda$, it is easy to show that there are always vectors $u \in \mathbf{R} \otimes \Lambda$ satisfying (3.9): an example is

$$
u_{e}=\frac{1}{2} \sum_{i=1}^{n} e_{i}^{2} e_{i}^{*},
$$

where $\left\{e_{i}\right\}$ is any basis of $\Lambda$ and $\left\{e_{i}^{*}\right\}$ is the corresponding dual basis, which satisfies $e_{i} \cdot e_{j}^{*}=\delta_{i j}$. For self-dual $\Lambda$, the solution $u$ to (3.9) is unique, modulo $\Lambda$. Moreover, for self-dual $\Lambda$ any $u$ satisfying (3.9) must necessarily obey $2 u \in \Lambda$ and has the norm

$$
u^{2} \equiv\left(n_{L}-n_{R}\right) / 4(\bmod 2)
$$

where $\left(n_{L}, n_{R}\right)$ is the signature of $\Lambda$ (eq.(3.10) is proved e.g. in [17]).

By no means is it suggested that these choices of $\Lambda, u, v$ are the most general possible to yield modular invariant $A^{u, v}(\Lambda)$ (that would be false), but they turn out to be sufficient to span any other choice.

For any (not necessarily positive) type $\mathcal{T}$, define

$$
\begin{aligned}
W Z_{\Lambda}^{u}(\mathcal{T})\left(z_{L} z_{R} \mid \tau\right) & \stackrel{\text { def }}{=} \exp \left[\pi i\left(n_{R}-n_{L}\right) / 2\right] \sum_{w_{L i}, w_{R j}} \epsilon\left(w_{L 1}\right) \cdots \epsilon\left(w_{R r}\right) \\
& \cdot \frac{A^{u, u}(\Lambda)\left(\sqrt{k_{L 1}+h_{L 1}^{\vee}} w_{L 1}\left(z_{L 1}\right), \ldots, \sqrt{k_{R r}+h_{R r}^{\vee}} w_{R r}\left(z_{R r}\right) \mid \tau\right)}{D\left(\mathcal{T}_{L}\right)\left(z_{L} \mid \tau\right) D\left(\mathcal{T}_{R}\right)\left(z_{R} \mid \tau\right)^{*}} \\
D\left(\mathcal{T}_{L}\right)\left(z_{L} \mid \tau\right) & \stackrel{\text { def }}{=} D_{g_{L 1}}\left(z_{L 1} \mid \tau\right) \cdots D_{g_{L l}}\left(z_{L l} \mid \tau\right)
\end{aligned}
$$

with a similar formula for $D\left(\mathcal{T}_{R}\right)$, where the Weyl automorphisms $w_{L i}, w_{R j}$ are as in (3.5), and $n_{L}=\sum n_{L i}, n_{R}=\sum n_{R j}$ (the factor in the front of (3.11a) is introduced to avoid awkward complex coefficients later).

The previous comments may be summarized in the following way:

Theorem A: Let $\Lambda$ be any self-dual gluing of $\Lambda(\mathcal{T})$. Let $u \in \mathbf{R} \otimes \Lambda$ be any vector satisfying eq.(3.9). Then the function $W Z_{\Lambda}^{u}(\mathcal{T})\left(z_{L} z_{R} \mid \tau\right)$ has the following properties:

(i) it can be written as a linear combination over $\mathbf{Z}$ of terms of the form (3.6);

(ii) let $u^{\prime} \in \mathbf{R} \otimes \Lambda$ be any other vector satisfying eq.(3.9). Then

$$
W Z_{\Lambda}^{u^{\prime}}(\mathcal{T})=(-1)^{\left(u-u^{\prime}\right)^{2}} W Z_{\Lambda}^{u}(\mathcal{T})
$$


(iii) $W Z_{\Lambda}^{u}(\mathcal{T})$ satisfies eq.(1.2a) iff

$$
\sum_{i=1}^{l} \frac{\rho_{L i}^{2}}{h_{L i}^{\vee}}-\sum_{j=1}^{r} \frac{\rho_{R j}^{2}}{h_{R j}^{\vee}} \equiv\left(n_{L}-n_{R}\right) / 4(\bmod 2) ;
$$

(iv) it satisfies eq.(1.2b) iff

$$
\sum_{i=1}^{l}\left\|\Delta_{+L i}\right\|-\sum_{j=1}^{r}\left\|\Delta_{+R j}\right\| \equiv n_{L}-n_{R}(\bmod 4) .
$$

This theorem defines a method for finding physical invariants of heterotic type $\mathcal{T}$. At least for small dimensions $n_{L}$ and $n_{R}$ and levels $k_{L i}, k_{R j}$, it is a straightforward task to find all self-dual gluings $\Lambda_{i}$ of $\Lambda(\mathcal{T})$. Then as with eq.(2.6), we search through the linear combinations of the resulting $W Z_{\Lambda_{i}}^{u_{i}}(\mathcal{T})$, to see when properties (P2) and (P3) are satisfied.

Note that we no longer require the self-dual lattice $\Lambda$ to be even. Thus we are freed from the severe restriction that $n_{L} \equiv n_{R}(\bmod 8)$. However we are now limited by eqs.(3.12).

When $\Lambda$ is even, $u=0$ may be chosen. Since $W Z_{\Lambda}^{0}(\{g, k\} ;\{g, k\})=W Z_{\Lambda}(g, k)$, we see then that this new method includes the RTW method as a special case. In fact, see Thm.D(i).

There is a second direction in which we can generalize the RTW method. Consider any positive type $\mathcal{T}^{+}$. Now consider any type $\mathcal{T}^{\prime}$ of the form

$$
\mathcal{T}^{\prime}=\left(\left\{g_{L 1}^{\prime}, 0\right\}, \ldots,\left\{g_{L l^{\prime}}^{\prime}, 0\right\} ;\left\{g_{R 1}^{\prime}, 0\right\}, \ldots,\left\{g_{R r^{\prime}}^{\prime}, 0\right\}\right),
$$

where either $l^{\prime}$ or $r^{\prime}$ may be 0 . Such a type, where all levels are 0 , will be called a null type. Let $\mathcal{T}=\mathcal{T}^{+}+\mathcal{T}^{\prime}$ denote the obvious combination of these types:

$$
\mathcal{T}^{+}+\mathcal{T}^{\prime}=\left(\left\{g_{L 1}, k_{L 1}\right\}, \ldots,\left\{g_{L l^{\prime}}^{\prime}, 0\right\} ;\left\{g_{R 1}, k_{R 1}\right\}, \ldots,\left\{g_{R r^{\prime}}^{\prime}, 0\right\}\right) .
$$

We say that $\mathcal{T}^{+}+\mathcal{T}^{\prime}$ is the augment of $\mathcal{T}^{+}$by $\mathcal{T}^{\prime}$. We are interested in choosing $\mathcal{T}^{\prime}$ so that $\mathcal{T}^{+}+\mathcal{T}^{\prime}$ satisfies eqs.(3.12), regardless of whether or not $\mathcal{T}^{+}$alone does. The idea is that because of (3.4), the function $W Z_{\Lambda}^{u}\left(\mathcal{T}^{+}+\mathcal{T}^{\prime}\right)$ for any self-dual gluing $\Lambda$ of $\Lambda\left(\mathcal{T}^{+}+\mathcal{T}^{\prime}\right)$ will be expressible as the sum of the terms in (3.5), and hence will actually be an invariant of type $\mathcal{T}^{+}$.

This will be discussed further in the following sections. This approach to construct physical invariants of type $\mathcal{T}^{+}$by taking linear combinations of the functions $W Z_{\Lambda}^{u}\left(\mathcal{T}^{+}+\right.$ $\mathcal{T}^{\prime}$ ), will be called the generalized $R T W$ method. Examples will be be given in Sec.5.

Before we leave this section, let us express this more precisely and introduce some useful notation. Consider any (not necessarily positive) type $\mathcal{T}$. Let $\mathcal{L}(\mathcal{T})$ be the set of all pairs $(\Lambda, u)$, where $\Lambda$ is a self-dual gluing of $\Lambda(\mathcal{T})$, and $\Lambda$, $u$ satisfies eq.(3.9). Let $\mathcal{L}_{e v}(\mathcal{T})$ consist of all even self-dual gluings of $\Lambda(\mathcal{T})$. There are finitely many lattices in both $\mathcal{L}(\mathcal{T})$ and $\mathcal{L}_{e v}(\mathcal{T}) ; \mathcal{L}_{e v}(\mathcal{T})$ will be empty unless $n_{L} \equiv n_{R}(\bmod 8)$. Finally, let $\Omega^{L}(\mathcal{T})$ be the space 
consisting of all linear combinations (over $\mathbf{C}$ ) of the functions $W Z_{\Lambda}^{u}(\mathcal{T}), \forall(\Lambda, u) \in \mathcal{L}(\mathcal{T})$, and let $\Omega_{e v}^{L}(\mathcal{T})$ be the space spanned by all functions $W Z_{\Lambda}^{0}(\mathcal{T})$, for $\Lambda \in \mathcal{L}_{e v}(\mathcal{T})$. We shall call these, respectively, the lattice commutant and even lattice commutant of type $\mathcal{T}$.

Now let $\mathcal{T}^{+}$be positive. By $\Omega_{W}\left(\mathcal{T}^{+}\right)$we mean the Weyl-folded commutant of type $\mathcal{T}^{+}$, i.e. the space of all modular invariant functions $Z\left(z_{L} z_{R} \mid \tau\right)$ of type $\mathcal{T}^{+}$. The generalized RTW method involves getting at the Weyl-folded commutant, and hence the physical invariants, of type $\mathcal{T}^{+}$by computing the (even) lattice commutant of augmented type $\mathcal{T}^{+}+\mathcal{T}^{\prime}$, for some null type $\mathcal{T}^{\prime}$. We will show in Sec.6 that the (even) lattice commutant of type $\mathcal{T}^{+}+\mathcal{T}^{\prime}$ either equals $\{0\}$, or equals $\Omega_{W}\left(\mathcal{T}^{+}\right)$; we will also show that for any positive $\mathcal{T}^{+}$there exist null types $\mathcal{T}^{\prime}, \mathcal{T}^{\prime \prime}$ such that $\Omega^{L}\left(\mathcal{T}^{+}+\mathcal{T}^{\prime}\right)=\Omega_{W}\left(\mathcal{T}^{+}\right)$and $\Omega_{e v}^{L}\left(\mathcal{T}^{+}+\mathcal{T}^{\prime \prime}\right)=$ $\Omega_{W}\left(\mathcal{T}^{+}\right)$; and we will characterize all $\mathcal{T}^{\prime}, \mathcal{T}^{\prime \prime}$ for which these equalities hold.

The RTW method, on the other hand, may be equated with the set $\Omega_{e v}^{L}\left(\mathcal{T}^{+}\right)$. For symmetric $\mathcal{T}^{+}$this will always equal the Weyl-folded commutant of type $\mathcal{T}^{+}$(see [9]), but for heterotic $\mathcal{T}^{+}$it usually will not.

\section{Analysis of the method}

This section begins the analysis of the completeness of the generalized RTW method which will be concluded in Sec.6. It also includes a condition (4.3) the type must satisfy in order that physical invariants of that type exist.

For the convenience of the reader, in the appendix is listed $\rho^{2}, h^{\vee},\left\|\Delta_{+}\right\|$and $M$ for each simple Lie algebra $g$. These quantities will be recurring throughout this section.

In the following theorem we make use of a relation called similarity, which is discussed in some detail in [18]. It can be defined in the following way. First, call two lattices $\Lambda, \Lambda^{\prime}$, with bases $\left\{\beta_{1}, \ldots, \beta_{n}\right\},\left\{\beta_{1}^{\prime}, \ldots, \beta_{n^{\prime}}^{\prime}\right\}$, rationally equivalent if their associated quadratic forms $Q\left(x_{1}, \ldots, x_{n}\right)=\left(\sum_{i} x_{i} \beta_{i}\right)^{2}$ and $Q^{\prime}\left(y_{1}, \ldots, y_{n^{\prime}}\right)=\left(\sum_{j} y_{j} \beta_{j}^{\prime}\right)^{2}$ are transformable into each other using linear maps $\vec{y}=A \vec{x}$ and $\vec{x}=B \vec{y}$, where all $A_{i j}, B_{i j} \in \mathbf{Q}$. Let $\Lambda_{1}$ and $\Lambda_{2}$ be two positive definite lattices of dimensions $n_{1}$ and $n_{2}$. Then we write $\Lambda_{1} \sim \Lambda_{2}$ iff $\Lambda_{1} \oplus I_{n_{2}}$ and $\Lambda_{2} \oplus I_{n_{1}}$ are rationally equivalent. (Here and throughout this paper, $I_{n}$ is the $n$-dimensional orthonormal lattice.) It turns out (see [18] for details) that for direct sums of the root lattices and their scalings, which is the case of interest here, similarities can easily be determined. The similarities of the coroot lattices are also included in the appendix below.

Theorem B: The lattice method given in Thm.A (i.e. taking linear combinations of $W Z_{\Lambda}^{u}(\mathcal{T})$ for generating physical invariants requires that the following conditions on the (not necessarily positive) type $\mathcal{T}=\left(\mathcal{T}_{L} ; \mathcal{T}_{R}\right)$ must be satisfied:

$$
\begin{gathered}
M\left(\mathcal{T}_{L}\right) \sim M\left(\mathcal{T}_{R}\right) ; \\
\sum_{i=1}^{l} \frac{\rho_{L i}^{2}}{h_{L i}^{\vee}}-\sum_{j=1}^{r} \frac{\rho_{R j}^{2}}{h_{R j}^{\vee}} \equiv\left(n_{L}-n_{R}\right) / 4(\bmod 2) ; \\
\sum_{i=1}^{l} \frac{\rho_{L i}^{2}}{h_{L i}^{\vee}+k_{L i}}-\sum_{j=1}^{r} \frac{\rho_{R j}^{2}}{h_{R j}^{\vee}+k_{R j}} \equiv\left(n_{L}-n_{R}\right) / 4(\bmod 2) .
\end{gathered}
$$


Thus eqs.(4.1) are necessary for the lattice commutant $\Omega^{L}(\mathcal{T})$ of type $\mathcal{T}$ to contain physical invariants. A (not necessarily positive) type $\mathcal{T}$ which satisfies eqs.(4.1) will be called an accessible type; $\mathcal{T}$ will be called weakly accessible if it satisfies eqs. $(4.1 a, b)$ but not necessarily $(4.1 c)$.

The condition in $(4.1 a)$ is precisely the statement [18] that the base lattice $\Lambda(\mathcal{T})$ has self-dual gluings, in other words that the set $\mathcal{L}(\mathcal{T})$ be nonempty. It implies among other things that the determinant $|\Lambda(\mathcal{T})|$ must be a perfect square.

Eq. (4.1b) is just (3.12a), and is required for $W Z_{\Lambda}^{u}(\mathcal{T})$ to be invariant under $\tau \rightarrow \tau+1$. By the "strange" formula of Freudenthal-de Vries (or by explicit calculation using the values given in the appendix) it can be seen that (3.12b) is satisfied whenever $(3.12 a)$ is. Thus $W Z_{\Lambda}^{u}(\mathcal{T})$ will be an invariant iff $(4.1 b)$ holds.

Eq. (4.1c) is the statement that the vector

$$
x_{u}=-u+\left(\frac{\rho_{L 1}}{\sqrt{k_{L 1}+h_{L 1}^{\vee}}}, \ldots ; \ldots, \frac{\rho_{R r}}{\sqrt{k_{R r}+h_{R r}^{\vee}}}\right)
$$

must satisfy (3.9). If (4.1c) were not satisfied, then the vector $x_{u}$ could lie in no $\Lambda$, where $(\Lambda, u) \in \mathcal{L}(\mathcal{T})$. That would mean that no $W Z_{\Lambda}^{u}(\mathcal{T})$, when expanded into terms of the form of $(3.3)$, would contain

$$
\chi_{0}\left(\mathcal{T}_{L}\right)\left(z_{L}, \tau\right) \cdot \chi_{0}\left(\mathcal{T}_{R}\right)\left(z_{R}, \tau\right)^{*}
$$

and so no linear combination could possibly satisfy property (P3).

To help us determine how overly restrictive eqs.(4.1) are, and hence how general the method described in Sec.3 actually is, we will now give a condition that the type of any physical invariant must satisfy.

Theorem C: Let $\mathcal{T}^{+}$be the type of some physical invariant. Then the following condition must be satisfied:

$$
\sum_{i=1}^{l} \frac{\rho_{L i}^{2}}{h_{L i}^{\vee}+k_{L i}}-\sum_{j=1}^{r} \frac{\rho_{R j}^{2}}{h_{R j}^{\vee}+k_{R j}} \equiv \sum_{i=1}^{l} \frac{\rho_{L i}^{2}}{h_{L i}^{\vee}}-\sum_{j=1}^{r} \frac{\rho_{R j}^{2}}{h_{R j}^{\vee}}(\bmod 2) .
$$

Eq.(4.3) follows from invariance under $\tau \rightarrow \tau+1$ (i.e. (1.2a)), and the requirement (see property (P3)) that the invariant of type $\mathcal{T}$ must contain the term in (4.2b) with nonzero coefficient. (It is proven in [10] — see also [14] — that the products in (3.6) for $\lambda_{L} \in P_{+}\left(\mathcal{T}_{L}\right)$ and $\lambda_{R} \in P_{+}\left(\mathcal{T}_{R}\right)$ are linearly independent. They are linearly independent even when, in the symmetric case considered by Roberts and Terao [5], we choose $z_{L}=z_{R}$ to be real.)

Again, it should be stressed that (4.3) must be satisfied by any type which is realized by a physical invariant; it is not assumed that that invariant is obtainable using the method introduced in Sec.3.

In the following section we will use Thm.C to help us find all heterotic physical invariants of total rank $n_{L}+n_{R}<4$. 
Note that a symmetric type automatically satisfies (4.3) (which is to be expected, since any symmetric type has at least one physical invariant, namely the diagonal one), and also satisfies eqs.(4.1), i.e. is also an accessible type.

For heterotic types, (4.3) is quite severe, but eqs.(4.1) are even more so. A heterotic type $\mathcal{T}^{+}$which has a physical invariant, need not be an accessible type. When $\mathcal{T}^{+}$is not accessible, its physical invariant(s) cannot be obtained as a linear combination of $W Z_{\Lambda}^{u}\left(\mathcal{T}^{+}\right)$. However, this is not a serious problem, for the following result can be shown:

Corollary $\mathbf{C}$ : Let $\mathcal{T}$ be any type.

(i) Then there exists a null type $\mathcal{T}^{\prime}$ such that the augment $\mathcal{T}+\mathcal{T}^{\prime}$ is weakly accessible.

(ii) Suppose there is a physical invariant of type $\mathcal{T}$. Then the augment $\mathcal{T}+\mathcal{T}^{\prime}$ in (i) will in addition be accessible.

We will show in Sec. 6 that the augment $\mathcal{T}+\mathcal{T}^{\prime}$ in (ii) necessarily satisfies $\Omega^{L}\left(\mathcal{T}+\mathcal{T}^{\prime}\right)=$ $\Omega_{W}(\mathcal{T})$. Incidently, there are infinitely many null types $\mathcal{T}^{\prime}$ that will work in (i) and (ii) (e.g. add arbitrary numbers of copies of $\left\{A_{1}, 0\right\}$ to either side of any $\mathcal{T}^{\prime}$ which works). The proof of (i) is not difficult, and (ii) is an immediate corollary of (i) and Thm.C.

The following result will simplify the completeness proof in Sec.6. It also is useful in practical calculations, as will be illustrated in the next section.

Theorem D: Let $\mathcal{T}$ be any (not necessarily positive) accessible type. Then

(i) if $n_{L} \equiv n_{R}(\bmod 8)$, then for odd $\Lambda$ with $(\Lambda, u) \in \mathcal{L}(\mathcal{T}), W Z_{\Lambda}^{u}(\mathcal{T})$ can be written as the difference $W Z_{\Lambda_{1}}^{0}(\mathcal{T})-W Z_{\Lambda_{2}}^{0}(\mathcal{T})$ for two even $\Lambda_{1}, \Lambda_{2} \in \mathcal{L}_{\text {ev }}(\mathcal{T})$

(ii) for $\mathcal{T}^{\prime}=\left(\left\{A_{1}, 0\right\}\right.$; ) or $\mathcal{T}^{\prime}=\left(;\left\{A_{1}, 0\right\}\right), \mathcal{T}+\mathcal{T}^{\prime}$ is also accessible, and for any $(\Lambda, u) \in \mathcal{L}\left(T+T^{\prime}\right), W Z_{\Lambda}^{u}\left(\mathcal{T}+\mathcal{T}^{\prime}\right)$ equals $c \cdot W Z_{\Lambda^{\prime}}^{u^{\prime}}(\mathcal{T})$, for some $\left(\Lambda^{\prime}, u^{\prime}\right) \in \mathcal{L}(\mathcal{T})$, and some constant $c$.

To prove Thm.D(i), write $\Lambda=\Lambda_{e} \cup\left(\Lambda_{e}+g\right)$, where $\Lambda_{e}$ equals the set of all evennormed vectors in $\Lambda$ and $g^{2}$ is odd. Then define the gluings $\Lambda_{1}=\Lambda_{e} \cup\left(\Lambda_{e}+u\right)$ and $\Lambda_{2}=\Lambda_{e} \cup\left(\Lambda_{e}+u+g\right)$. The proof of (ii) is similar but a little longer. First note $A_{2}^{(2) *} / A_{2}^{(2)}$ consists of 4 cosets: [0] $\stackrel{\text { def }}{=} 2 \mathbf{Z}$; [1] $\stackrel{\text { def }}{=} \frac{1}{2}+2 \mathbf{Z}$; [2] $\stackrel{\text { def }}{=} 1+2 \mathbf{Z}$; and [3] $\stackrel{\text { def }}{=} \frac{3}{2}+2 \mathbf{Z}$, using obvious notation. Write the cosets $x \in \Lambda /\left(A_{2}^{(2)} \oplus \Lambda(\mathcal{T})\right)$ as $\left[x_{1}, x_{2}\right]$, where $x_{1}=0,1,2,3$ and $x_{2} \in \Lambda(\mathcal{T})^{*} / \Lambda(\mathcal{T})$. case 1: If $[2,0] \subset \Lambda$ then $\Lambda=\mathbf{Z} \oplus \Lambda^{\prime}$, where $\Lambda^{\prime}$ is a self-dual gluing of $\Lambda(\mathcal{T})$. Write $u=\left(u_{1}, u^{\prime}\right)$, then $W Z_{\Lambda}^{u}\left(\mathcal{T}+\mathcal{T}^{\prime}\right)=2 \cdot W Z_{\Lambda^{\prime}}^{u^{\prime}}(\mathcal{T})$. case 2: Otherwise, $\exists g \in \Lambda(\mathcal{T})^{*} / \Lambda(\mathcal{T})$ such that $[1, g] \subset \Lambda$. Without loss of generality (see Thm.A(ii)) take $u=\left(0, u_{2}\right)$. Write $\Lambda_{2}=\left\{x_{2} \in \Lambda(\mathcal{T})^{*} \mid\left(0, x_{2}\right) \in \Lambda\right\}$. Then $\left|\Lambda_{2}\right|=4$ and $\Lambda_{2}$ is integral. The order of $g$ in $\Lambda_{2}$ is 4 (since [1] has order 4). case 2a: If $\Lambda_{2}$ is an even lattice, then take $\Lambda^{\prime}=\Lambda_{2} \cup\left(\Lambda_{2}+2 g\right)$ and $u^{\prime}=u_{2}+g$. case $2 b$ : Otherwise there is a vector $g^{\prime} \in \Lambda_{2}$ with odd norm. Then take $\Lambda^{\prime}=\Lambda_{2} \cup\left(\Lambda_{2}+2 g\right)$ and $u^{\prime}=u_{2}+g^{\prime}-g$.

Note that there are no conditions on $n_{L}, n_{R}$ in Thm.D(ii), so the same conclusion holds for any $\mathcal{T}^{\prime}=\left(\left\{A_{1}, 0\right\}, \ldots,\left\{A_{1}, 0\right\} ;\left\{A_{1}, 0\right\}, \ldots,\left\{A_{1}, 0\right\}\right)$. Thm.D $(\mathrm{i})$ says that whenever $n_{L} \equiv n_{R}(\bmod 8), \Omega_{e v}^{L}(\mathcal{T})=\Omega^{L}(\mathcal{T})$. Of course, for $\mathcal{T}$ symmetric this forced by the completeness proof in $[9]$. 


\section{Examples: the physical invariants of low rank}

In this section we will work out some explicit examples to help illustrate this method. In particular we will find all heterotic physical invariants of total rank $n_{L}+n_{R}<4$.

Let us first "solve" (4.3) for the types of smallest total rank. First consider positive types of total rank 1 . These will look like $\mathcal{T}^{+}=\left(\left\{A_{1}, k\right\} ;\right)$ or equivalently $\left(;\left\{A_{1}, k\right\}\right)$, for some $k>0$. From the appendix we can write (4.3) as

$$
\frac{\frac{1}{2}}{k+2} \equiv \frac{\frac{1}{2}}{2}(\bmod 2),
$$

i.e. $k \equiv 0(\bmod 8(k+2))$. This has no solution for $k>0$. Thus Thm.C tells us that there can be no physical invariant of total rank 1 .

Similar but lengthier arguments apply to the other ranks. In particular it can be shown that the only types of total rank 2 which satisfy (4.3) are the symmetric types $\left(\left\{A_{1}, k\right\} ;\left\{A_{1}, k\right\}\right)$. There are exactly 6 solutions of total rank 3 :

$$
\begin{aligned}
\mathcal{T}_{I} & =\left(\left\{A_{2}, 1\right\} ;\left\{A_{1}, 4\right\}\right) \\
\mathcal{T}_{I I} & =\left(\left\{A_{1}, 1\right\},\left\{A_{1}, 1\right\} ;\left\{A_{1}, 4\right\}\right) \\
\mathcal{T}_{I I I} & =\left(\left\{C_{2}, 1\right\} ;\left\{A_{1}, 10\right\}\right) \\
\mathcal{T}_{I V} & =\left(\left\{G_{2}, 1\right\} ;\left\{A_{1}, 28\right\}\right) \\
\mathcal{T}_{V} & =\left(\left\{A_{1}, 1\right\},\left\{A_{1}, 2\right\} ;\left\{A_{1}, 10\right\}\right) \\
\mathcal{T}_{V I} & =\left(\left\{A_{1}, 3\right\},\left\{A_{1}, 1\right\} ;\left\{A_{1}, 28\right\}\right) .
\end{aligned}
$$

All of these are inaccessible types - e.g. $\mathcal{T}_{I}$ fails to satisfy any of eqs.(4.1). However the augments

$$
\begin{aligned}
\mathcal{T}_{I}+\mathcal{T}_{I}^{\prime} & =\left(\left\{A_{2}, 1\right\},\left\{C_{2}, 0\right\} ;\left\{A_{1}, 4\right\},\left\{A_{3}, 0\right\}\right) \\
\mathcal{T}_{I I}+\mathcal{T}_{I I}^{\prime} & =\left(\left\{A_{1}, 1\right\},\left\{A_{1}, 1\right\},\left\{G_{2}, 0\right\},\left\{F_{4}, 0\right\} ;\left\{A_{1}, 4\right\}\right) \\
\mathcal{T}_{I I I}+\mathcal{T}_{I I I}^{\prime} & =\left(\left\{C_{2}, 1\right\},\left\{G_{2}, 0\right\} ;\left\{A_{1}, 10\right\},\left\{C_{3}, 0\right\}\right) \\
\mathcal{T}_{I V}+\mathcal{T}_{I V}^{\prime} & =\left(\left\{G_{2}, 1\right\},\left\{C_{2}, 0\right\} ;\left\{A_{1}, 28\right\},\left\{B_{3}, 0\right\}\right) \\
\mathcal{T}_{V}+\mathcal{T}_{V}^{\prime} & =\left(\left\{A_{1}, 1\right\},\left\{A_{1}, 2\right\},\left\{A_{3}, 0\right\} ;\left\{A_{1}, 10\right\},\left\{D_{5}, 0\right\}\right) \\
\mathcal{T}_{V I}+\mathcal{T}_{V I}^{\prime} & =\left(\left\{A_{1}, 3\right\},\left\{A_{1}, 1\right\} ;\left\{A_{1}, 28\right\},\left\{A_{2}, 0\right\},\left\{D_{7}, 0\right\}\right)
\end{aligned}
$$

are all accessible.

It can be shown (this will be discussed below) that there is exactly one physical invariant of types $\mathcal{T}_{I}, \mathcal{T}_{I I I}, \mathcal{T}_{I V}$, and $\mathcal{T}_{V I}$, and none of types $\mathcal{T}_{I I}$ and $\mathcal{T}_{V}$. The rank 3 physical invariants are:

$$
\begin{aligned}
Z_{I} & =\chi_{00} \chi_{0}^{*}+\chi_{00} \chi_{4}^{*}+\chi_{10} \chi_{2}^{*}+\chi_{01} \chi_{2}^{*} \\
Z_{I I I} & =\chi_{00} \chi_{0}^{*}+\chi_{00} \chi_{6}^{*}+\chi_{10} \chi_{3}^{*}
\end{aligned}
$$




$$
\begin{aligned}
& +\chi_{10} \chi_{7}^{*}+\chi_{01} \chi_{4}^{*}+\chi_{01} \chi_{10}^{*} \\
Z_{I V}= & \chi_{00} \chi_{0}^{*}+\chi_{01} \chi_{6}^{*}+\chi_{00} \chi_{10}^{*}+\chi_{01} \chi_{12}^{*} \\
& +\chi_{01} \chi_{16}^{*}+\chi_{00} \chi_{18}^{*}+\chi_{01} \chi_{22}^{*}+\chi_{00} \chi_{28}^{*} \\
Z_{V I}=\{ & \left\{\chi_{0} \chi_{0}+\chi_{3} \chi_{1}\right\} \cdot\left\{\chi_{0}^{*}+\chi_{10}^{*}+\chi_{18}^{*}+\chi_{28}^{*}\right\} \\
& +\left\{\chi_{1} \chi_{1}+\chi_{2} \chi_{0}\right\} \cdot\left\{\chi_{6}^{*}+\chi_{12}^{*}+\chi_{16}^{*}+\chi_{22}^{*}\right\},
\end{aligned}
$$

where in the subscripts we used the Dynkin labels to represent the weights $\lambda$, and the superscripts ' $g, k$ ' have been dropped.

Thus the physical invariants of total rank $<4$ have been completely classified; eqs. (5.3) contain the only heterotic ones, and [4] enumerated all the symmetric ones, which are of type $\left(\left\{A_{1}, k\right\} ;\left\{A_{1}, k\right\}\right)$ (and total rank=2).

We will now use the generalized RTW method developed in Sec.3 to construct the physical invariant $Z_{I}$ in $(5.3 a)$. The other physical invariants in (5.3) can be constructed similarly.

Consider $\mathcal{T}_{I}$. Because $\mathcal{T}_{I}$ does not satisfy $(4.1 a), \mathcal{L}\left(\mathcal{T}_{I}\right)=\{\}$ and $\Omega^{L}\left(\mathcal{T}_{I}\right)=\{0\}$. That is one of the possible consequences of the type being inaccessible, and is the reason that we must turn to the accessible augment $\mathcal{T}_{I}^{\prime \prime}=\mathcal{T}_{I}+\mathcal{T}_{I}^{\prime}$ given in (5.2a). We will construct the desired self-dual lattice using gluing; for a review of the gluing theory of lattices, see $[13,18]$.

The base lattice is $\Lambda\left(\mathcal{T}_{I}^{\prime \prime}\right)=\left(A_{2}^{(4)} \oplus\left(A_{1} \oplus A_{1}\right)^{(3)} ; A_{1}^{(6)} \oplus A_{3}^{(4)}\right)$, which can also be written $\left(A_{2}^{(4)}, I_{1}^{(6)}, I_{1}^{(6)} ; I_{1}^{(12)}, A_{3}^{(4)}\right)$ using obvious notation. This has the determinant $\left|\Lambda\left(\mathcal{T}_{I}^{\prime \prime}\right)\right|=$ $4^{8} \cdot 3^{4}$, so in order for $\Lambda$ to be a self-dual gluing, the glue group $\Lambda / \Lambda\left(T_{I}^{\prime \prime}\right)$ must have order $\sqrt{4^{8} \cdot 3^{4}}=2^{8} \cdot 3^{2}=2304$.

A convenient basis for the dual $\left(A_{2}^{(4)}\right)^{*}$ consists of the scaled fundamental weight $\beta_{1} / 2$ and the scaled simple root $\alpha_{1} / 2$. Let $\lambda_{m, n}$ denote the vector $m \beta_{1} / 2+n \alpha_{1} / 2$. Then $\lambda_{m, n} \in A_{2}^{(4)}$ iff $m \equiv 0(\bmod 12)$ and $n \equiv 0(\bmod 4)$. In this basis, $\rho / 2=\lambda_{3,-1}$.

Similarly, a convenient parametrization of $\left(A_{3}^{(4)}\right)^{*}$ is $\mu_{p, q, r}=p \beta_{1} / 2+q \alpha_{1} / 2+r \alpha_{2} / 2$. $\mu_{p, q, r} \in A_{3}^{(4)}$ iff $p \equiv 0(\bmod 16)$ and $q \equiv r \equiv 0(\bmod 4)$. The $\rho / 2$ here equals $\mu_{6,1,3}$.

Finally, $\left(I_{1}^{(6)}, I_{1}^{(6)} ; I_{1}^{12)}\right)^{*}$ can be parametrized by the triples $(a, b, c)$ corresponding to the vector $(a / \sqrt{6}, b / \sqrt{6} ; c / \sqrt{12})$. That vector lies in $\left(I_{1}^{(6)}, I_{1}^{(6)} ; I_{1}^{(12)}\right)$ iff $a \equiv b \equiv 0(\bmod 6)$ and $c \equiv 0(\bmod 12)$. The scaled $\rho$ for $\left(C_{2}^{(3)} ; A_{1}^{(6)}\right)$ then is given by the triple $(2,1 ; 1)$.

Note that by Thm.D(i) we may restrict our attention to even self-dual gluings $\Lambda$, since $n_{L}=n_{R}=4$. Because we want to find an invariant satisfying (P3), we may as well include the vector $x_{0}$ in $(4.2 a)$ as a glue vector: hence our first glue will be $g_{1}=$ $\left(\lambda_{3,-1}, 2,1 ; 1, \mu_{613}\right)$. This glue has order 24 . Of course it has even norm - this is implied by $(4.1 c)$. For our second glue we may choose any $g_{2}$ for which $g_{2} \cdot g_{1} \in \mathbf{Z}$ and $g_{2}^{2} \in 2 \mathbf{Z}$; an example is $g_{2}=\left(\lambda_{10}, 1,1 ; 0, \mu_{003}\right)$, which has order 12 . Similarly, $g_{3}=(0,3,3 ; 6,0)$ and $g_{4}=\left(\lambda_{01}, 0,0 ; 0, \mu_{010}\right)$ have even norms and integral dot products with all other glues. Their orders are 2 and 4 , respectively.

Choosing $g_{1}, g_{2}, g_{3}, g_{4}$ as our glue generators, it is easy to see that the group generated 
by them $\left(\bmod \Lambda\left(\mathcal{T}_{I}^{\prime \prime}\right)\right)$ has 2304 elements. Thus the gluing

$$
\Lambda=\Lambda\left(\mathcal{T}_{I}^{\prime \prime}\right)\left[g_{1}, g_{2}, g_{3}, g_{4}\right] \stackrel{\text { def }}{=} \bigcup_{a, b, c, d \in \mathbf{Z}}\left(a g_{1}+b g_{2}+c g_{3}+d g_{4}+\Lambda\left(\mathcal{T}_{I}^{\prime \prime}\right)\right)
$$

is even and self-dual, so lies in $\mathcal{L}_{e v}\left(\mathcal{T}_{I}^{\prime \prime}\right)$. It turns out to have the partition function $W Z_{\Lambda}^{0}\left(\mathcal{T}_{I}^{\prime \prime}\right)=48 Z_{I}$, and thus directly gives us one of the physical invariants.

There are many different ways to show that eqs.(5.3) exhaust all the physical invariants of type $\mathcal{T}_{I}, \ldots, \mathcal{T}_{V I}$, including finding all relevant lattice gluings (this is much easier than it sounds, thanks to the large Weyl groups involved). For types of such small total rank and levels, the direct approach of explicitly calculating the $S$ and $T$ matrices corresponding to the modular transformations $\tau \rightarrow \tau+1$ and $\tau \rightarrow-1 / \tau$ and finding all combinations of the characters invariant under them also will work fine, but becomes totally unfeasible in more complicated cases.

Incidently, refs.[5] both use the shift lattice construction method. For the heterotic case (and perhaps also for the symmetric case), the gluing method used above seems more efficient.

The four heterotic physical invariants obtained in (5.3) can also be found using the technique of conformal embeddings [8]. For example, (5.3a) comes from the level 1 diagonal invariant of $A_{2}$. However, the lattice method found here is more general, and we can expect it to reveal more solutions than conformal embeddings for higher rank (see Thm.E below).

The accessible types of smallest total rank which satisfy (4.3) include

$$
\begin{aligned}
\mathcal{T}_{i} & =\left(\left\{B_{4}, 14\right\} ;\right) \\
\mathcal{T}_{i i} & =\left(\left\{C_{4}, 10\right\} ;\right) .
\end{aligned}
$$

Both of these are of total rank 4. It is not difficult to write down the other heterotic rank 4 solutions to (4.3): there are (unfortunately) infinitely many of type

$$
\left(\left\{A_{1}, k\right\},\left\{A_{1}, k^{\prime}\right\} ;\left\{A_{1}, k^{\prime \prime}\right\},\left\{A_{1}, k^{\prime \prime \prime}\right\}\right),
$$

and approximately 50 others.

In the symmetric case, this lattice method is currently being used in a computer program by Q. Ho-Kim [19] to find all symmetric physical invariants of total rank 4, and levels up to around 35 .

\section{The proof of completeness}

In this section we prove the completeness of the generalized $R T W$ lattice method. Much of the notation used below is defined at the end of Sec.3.

In particular, consider any positive type $\mathcal{T}^{+}$. Let $\mathcal{T}^{\prime}$ be any null type for which the augment $\mathcal{T}=\mathcal{T}^{+}+\mathcal{T}^{\prime}$ is weakly accessible (see Cor.C(i)). That means that $\mathcal{T}$ satisfies

both eqs. $(4.1 a, b)$. The first of these says that the lattice set $\mathcal{L}(\mathcal{T})$ is nonempty, and the second says that for any $(\Lambda, u) \in \mathcal{L}(\mathcal{T})$, the function $W Z_{\Lambda}^{u}(\mathcal{T})$ is modular invariant. It 
can be shown (see the proof of Thm.7.8 in [18]) that $\mathcal{L}_{e v}(\mathcal{T})$ is nonempty iff both $(4.1 a)$ and $n_{L}+n_{L}^{\prime} \equiv n_{R}+n_{R}^{\prime}(\bmod 8)$ hold. In that case we also have that for all $\Lambda \in \mathcal{L}_{\text {ev }}(\mathcal{T})$, $W Z_{\Lambda}^{0}(\mathcal{T})$ is modular invariant.

Theorem E: Consider any positive type $\mathcal{T}^{+}$. Let $\mathcal{T}^{\prime}$ be any null type for which the augment $\mathcal{T}^{+}+\mathcal{T}^{\prime}$ is weakly accessible. Then the lattice commutant of type $\mathcal{T}^{+}+\mathcal{T}^{\prime}$ equals the Weyl-folded commutant of type $\mathcal{T}^{+}$:

$$
\Omega^{L}\left(\mathcal{T}^{+}+\mathcal{T}^{\prime}\right)=\Omega_{W}\left(\mathcal{T}^{+}\right)
$$

Moreover, when the ranks satisfy $n_{L}+n_{L}^{\prime} \equiv n_{R}+n_{R}^{\prime}(\bmod 8)$, the even lattice commutant equals the Weyl-folded commutant:

$$
\Omega_{\text {ev }}^{L}\left(\mathcal{T}^{+}+\mathcal{T}^{\prime}\right)=\Omega_{W}\left(\mathcal{T}^{+}\right)
$$

Hence this says that the generalized RTW method succeeds in generating all (heterotic) invariants, and in particular all physical ones. Although it holds for any null type $\mathcal{T}^{\prime}$ for which $\mathcal{T}^{+}+\mathcal{T}^{\prime}$ is weakly accessible, clearly it is most advantageous to choose one of smallest rank, and if possible one for which $n_{L}+n_{L}^{\prime} \equiv n_{R}+n_{R}^{\prime}(\bmod 8)$ also holds. Of course, if $\mathcal{T}^{+}$is already weakly accessible, then $\mathcal{T}^{\prime}$ may be taken to be empty: $\mathcal{T}^{\prime}=(;)$.

In [9] we previously showed that the RTW lattice method spans the commutant for symmetric $\mathcal{T}$. That argument was inspired by the analysis of [20]. As much as possible, we will try in the following proof to stick as close as possible to the machinery and notation developed in [9].

Note that Thm.A gives the containment $\Omega^{L} \subseteq \Omega_{W}$. To prove Thm.E, we will augment the type $\mathcal{T}^{+}+\mathcal{T}^{\prime}$ by some copies of $\left\{A_{1}, 0\right\}$. This will allow us to consider the simpler case of even self-dual lattices; by Thm.D, nothing is lost by using the resulting $\mathcal{T}_{h}$ instead of $\mathcal{T}^{+}+\mathcal{T}^{\prime}$. Most of the proof is devoted to establishing a mapping, given in eqs.(6.7), between the invariants of $\mathcal{T}_{h}$ and those of a certain symmetric type $\mathcal{T}_{s}$. The claim found below shows that each lattice partition function of $\mathcal{T}_{s}$ gets sent by our mapping to a lattice partition function of $\mathcal{T}_{h}$. We know [9] lattice partition functions span the commutant of $\mathcal{T}_{s}$, and using our (linear, surjective) mapping we then get that the lattice partition functions span that of $\mathcal{T}_{h}$.

Proof of Thm.E Choose any number $n_{L}^{\prime \prime} \geq 0$ for which $n_{L}+n_{L}^{\prime}+n_{L}^{\prime \prime} \equiv n_{R}+n_{R}^{\prime}(\bmod$ 8), and let $\mathcal{T}^{\prime \prime}=\left(\left\{A_{1}, 0\right\}^{n_{L}^{\prime \prime}} ;\right)$ be the null type consisting of $n_{L}^{\prime \prime}$ copies of $\left\{A_{1}, 0\right\}$. By Thm.D(ii), $\mathcal{T}_{h} \stackrel{\text { def }}{=} \mathcal{T}^{+}+\mathcal{T}^{\prime}+\mathcal{T}^{\prime \prime}$ will also be weakly accessible. Thanks to Thm.D, it will be more convenient to work with $\mathcal{T}_{h}$ than $\mathcal{T}^{+}+\mathcal{T}^{\prime}$. We will return to $\mathcal{T}^{+}+\mathcal{T}^{\prime}$ in the final paragraph of the proof.

Write $\mathcal{T}_{h}=\left(\mathcal{T}_{L} ; \mathcal{T}_{R}\right)$. Our first goal will be to establish a connection (see eqs.(6.7)) between invariants of the symmetric type $\mathcal{T}_{s} \stackrel{\text { def }}{=}\left(\mathcal{T}_{L} ; T_{L}\right)+\left(\mathcal{T}_{R} ; \mathcal{T}_{R}\right)$, and invariants of the heterotic type $\mathcal{T}_{h}$. That will permit us to exploit the result from [9] that lattice partition functions span the commutants of symmetric types. 
As was done in $[9,20]$, we will consider instead the commutant built up from the theta series of glue classes, rather than the one built up from characters. The latter can be recovered from the former by Weyl-folding, i.e. summing over the Weyl group as in the numerator of $(2.2 a)$. The details will be made clear below.

Define $\Lambda_{L}=\Lambda\left(\mathcal{T}_{L}\right)$ and $\Lambda_{R}=\Lambda\left(\mathcal{T}_{R}\right)$, so that $\Lambda\left(\mathcal{T}_{h}\right)=\left(\Lambda_{L} ; \Lambda_{R}\right)$. For each $\lambda_{L}=$ $\left(\lambda_{L 1}, \ldots, \lambda_{L n_{L}^{\prime \prime}}^{\prime \prime}\right) \in \Lambda_{L}^{*} / \Lambda_{L}$ and $z_{L}=\left(z_{L 1}, \ldots, z_{L n_{L}^{\prime \prime}}^{\prime \prime}\right) \in \mathbf{C} \otimes \Lambda_{L}$, define the function

$$
\begin{aligned}
s_{\lambda_{L}}\left(\mathcal{T}_{L}\right)\left(z_{L} \mid \tau\right) & \stackrel{\text { def }}{=}\left(\prod_{i=1}^{l} \frac{\Theta\left(\lambda_{L i}+M_{g_{L i}}^{\left(k_{L i}+h_{L i}^{\vee}\right)}\right)\left(\sqrt{k_{L i}+h_{L i}^{\vee}} z_{L i} \mid \tau\right)}{D_{g_{L i}}\left(z_{L i} \mid \tau\right)}\right) \cdot \\
& \left(\prod_{j=1}^{l^{\prime}} \frac{\Theta\left(\lambda_{L j}^{\prime}+M_{L j}^{\prime}{ }^{\left(h_{L j}^{\vee}{ }^{\prime}\right)}\right)\left(\sqrt{h_{L j}^{\vee}{ }^{\prime}} z_{L j}^{\prime} \mid \tau\right)}{D_{g_{L j}{ }^{\prime}}\left(z_{L j}^{\prime} \mid \tau\right)}\right) \cdot\left(\prod_{\ell=1}^{n_{L}^{\prime \prime}} \frac{\Theta\left(\lambda_{L \ell}^{\prime \prime}+A_{1}^{(2)}\right)\left(\sqrt{2} z_{L \ell}^{\prime \prime} \mid \tau\right)}{D_{A_{1}}\left(z_{L \ell}^{\prime \prime} \mid \tau\right)}\right)
\end{aligned}
$$

and for $\lambda_{R} \in \Lambda_{R}^{*} / \Lambda_{R}, z_{R} \in \mathbf{C} \otimes \Lambda_{R}$ define a function $t_{\lambda_{R}}\left(\mathcal{T}_{R}\right)\left(z_{R} \mid \tau\right)$ similarly. All terms $s_{\lambda_{L}}\left(\mathcal{T}_{L}\right) t_{\lambda_{R}}\left(\mathcal{T}_{R}\right)^{*}$ are linearly independent (this follows from Thm.4.5 in [14]).

Define $\Omega_{t h}\left(\mathcal{T}_{h}\right)$ to be the space of all modular invariant linear combinations

$$
Z=\sum N_{\lambda_{L} \lambda_{R}} s_{\lambda_{L}}\left(\mathcal{T}_{L}\right) t_{\lambda_{R}}\left(\mathcal{T}_{R}\right)^{*}
$$

where the sum is over all $\lambda_{L} \in \Lambda_{L}^{*} / \Lambda_{L}, \lambda_{R} \in \Lambda_{R}^{*} / \Lambda_{R}$. We will call these theta-invariants of type $\mathcal{T}_{h}$ to distinguish them from the character-invariants considered elsewhere in this paper. $N$ will be called the coefficient matrix of $Z$; its dimensions are $\left|\Lambda_{L}\right| \times\left|\Lambda_{R}\right|$, where as usual we denote determinants of lattices by $|\cdot|$.

There exist matrices $T_{L}, T_{R}, S_{L}$ and $S_{R}$ which describe how $s_{\lambda_{L}}\left(\mathcal{T}_{L}\right)$ and $t_{\lambda_{R}}\left(\mathcal{T}_{R}\right)$, respectively, transform under $\tau \rightarrow \tau+1$ and $\tau \rightarrow-1 / \tau$. They are explicitly given in [9]. Here it suffices to remark that they are symmetric and unitary. The function $Z$ in (6.2) is modular invariant iff both

$$
\begin{aligned}
& T_{L}^{\dagger} N T_{R}=N, \text { and } \\
& S_{L}^{\dagger} N S_{R}=N .
\end{aligned}
$$

From the comments made at the beginning of this section, we know $\mathcal{L}_{e v}\left(\mathcal{T}_{h}\right)$ is nonempty, so there exists an even self-dual gluing $\Lambda_{0}$ of $\left(\Lambda_{L} ; \Lambda_{R}\right)$ for which $W Z_{\Lambda_{0}}^{0}\left(\mathcal{T}_{h}\right)$ is a modular invariant. In fact, an identical calculation shows that the theta series of $\Lambda_{0}$, divided by the usual $D\left(\mathcal{T}_{L}\right) D\left(\mathcal{T}_{R}\right)^{*}$, also is modular invariant. We will denote it by $Z_{\Lambda_{0}}\left(\mathcal{T}_{h}\right)$ : writing it out explicitly, we get

$$
Z_{\Lambda_{0}}\left(\mathcal{T}_{h}\right)\left(z_{L} z_{R} \mid \tau\right)=\sum_{\left(\lambda_{L} ; \lambda_{R}\right) \in \Lambda_{0} /\left(\Lambda_{L} ; \Lambda_{R}\right)} s_{\lambda_{L}}\left(\mathcal{T}_{L}\right)\left(z_{L} \mid \tau\right) t_{\lambda_{R}}\left(\mathcal{T}_{L}\right)\left(z_{R} \mid \tau\right)^{*}
$$

It is in the form of (6.2) with coefficient matrix $N_{\Lambda_{0}}$ given by

$$
\left(N_{\Lambda_{0}}\right)_{\lambda_{L} \lambda_{R}}=\left\{\begin{array}{cc}
1 & \text { if }\left(\lambda_{L} ; \lambda_{R}\right) \in \Lambda_{0} \\
0 & \text { otherwise }
\end{array}\right.
$$


Hence $Z_{\Lambda_{0}}\left(\mathcal{T}_{h}\right)$ is a theta-invariant lying in $\Omega_{t h}\left(\mathcal{T}_{h}\right)$.

Let $Z_{L}, Z_{R}$ with coefficient matrices $N_{L}, N_{R}$ be any theta-invariants of type $\left(\mathcal{T}_{L} ; \mathcal{T}_{L}\right)$ and $\left(\mathcal{T}_{R} ; \mathcal{T}_{R}\right)$, respectively. Note that by $(6.3), N_{L} N_{\Lambda_{0}} N_{R}$ will be the coefficient matrix for a theta-invariant of type $\mathcal{T}_{h}=\left(\mathcal{T}_{L} ; \mathcal{T}_{R}\right)$. This observation motivates the following discussion, designed to establish the connection between $\mathcal{T}_{h}$ and $\mathcal{T}_{s}$ given in eqs.(6.7) below.

For any $\lambda_{L}, \lambda_{L}^{\prime} \in \Lambda_{L}^{*} / \Lambda_{L}$, let $N_{L}^{\lambda_{L} \lambda_{L}^{\prime}}\left(\mathcal{T}_{L}\right)$ denote the coefficient matrix defined by:

$$
\frac{A^{\left(\lambda_{L} ; 0\right),\left(\lambda_{L}^{\prime} ; 0\right)}\left(\Lambda_{L}^{D}\right)}{D\left(\mathcal{T}_{L}\right) D\left(\mathcal{T}_{L}\right)^{*}}=\sum_{\mu, \mu^{\prime} \in \Lambda_{L}^{*} / \Lambda_{L}}\left(N_{L}^{\lambda_{L} \lambda_{L}^{\prime}}\left(\mathcal{T}_{L}\right)\right)_{\mu \mu^{\prime}} s_{\mu}\left(\mathcal{T}_{L}\right) s_{\mu^{\prime}}\left(\mathcal{T}_{L}\right)^{*}
$$

where the function $A^{-,-}(\Lambda)$ on the LHS is given by (3.8a) above, and where $\Lambda_{L}^{D}$ denotes the diagonal gluing of $\left(\Lambda_{L} ; \Lambda_{L}\right)$ - i.e. the even, self-dual lattice

$$
\Lambda_{L}^{D} \stackrel{\text { def }}{=} \bigcup_{\lambda \in \Lambda_{L}^{*} / \Lambda_{L}}(\lambda ; \lambda)+\left(\Lambda_{L} ; \Lambda_{L}\right)
$$

Define the analogous matrix $N_{R}^{\lambda_{R} \lambda_{R}^{\prime}}\left(\mathcal{T}_{R}\right)$ similarly. The dimensions of these two complex matrices are $\left|\Lambda_{L}\right| \times\left|\Lambda_{L}\right|$ and $\left|\Lambda_{R}\right| \times\left|\Lambda_{R}\right|$, respectively.

Now choose any $k_{L}, k_{L}^{\prime} \in\left(\Lambda_{L}^{(2)}\right)^{*} / \Lambda_{L}^{(2)}$ and $k_{R}, k_{R}^{\prime} \in\left(\Lambda_{R}^{(2)}\right)^{*} / \Lambda_{R}^{(2)}$. Define the matrix $\left\{k_{L}, k_{R}, k_{L}^{\prime}, k_{R}^{\prime}\right\}_{h}$ by the matrix product

$$
\begin{aligned}
\left\{k_{L}, k_{R}, k_{L}^{\prime}, k_{R}^{\prime}\right\}_{h}= & \exp \left[-2 \pi i\left(k_{L} \cdot k_{L}^{\prime}+k_{R} \cdot k_{R}^{\prime}\right)\right] \cdot \\
& \cdot N_{L}^{\sqrt{2} k_{L}, \sqrt{2} k_{L}^{\prime}}\left(\mathcal{T}_{L}\right) N_{\Lambda_{0}} N_{R}^{\sqrt{2} k_{R}, \sqrt{2} k_{R}^{\prime}}\left(\mathcal{T}_{R}\right),
\end{aligned}
$$

where by ' $\sqrt{2} k_{L}$ ', etc. we mean the coset $\sqrt{2} k_{L}+\Lambda_{L} \in \Lambda_{L}^{*} / \Lambda_{L}$. Because $N_{\Lambda_{0}}$ is nonzero, an easy argument (see eq.(3.7b) in [9]) shows these $\{\cdots\}_{h}$ span the space of complex $\left|\Lambda_{L}\right| \times\left|\Lambda_{R}\right|$ matrices (though they are not linearly independent). Moreover, from (3.8b) we read off

$$
\begin{aligned}
& T_{L}^{\dagger}\left\{k_{L}, k_{R}, k_{L}^{\prime}, k_{R}^{\prime}\right\}_{h} T_{R}=\left\{k_{L}, k_{R}, k_{L}^{\prime}+k_{L}, k_{R}^{\prime}+k_{R}\right\}_{h}, \\
& S_{L}^{\dagger}\left\{k_{L}, k_{R}, k_{L}^{\prime}, k_{R}^{\prime}\right\}_{h} S_{R}=\left\{k_{L}^{\prime}, k_{R}^{\prime},-k_{L},-k_{R}\right\}_{h} .
\end{aligned}
$$

Note that eqs. $(6.6 b, c)$ are precisely the form obtained in the symmetric types analysis of [9]. In particular, define

$$
\left\{k_{L}, k_{R}, k_{L}^{\prime}, k_{R}^{\prime}\right\}_{s}=\exp \left[-2 \pi i\left(k_{L} \cdot k_{L}^{\prime}+k_{R} \cdot k_{R}^{\prime}\right)\right] N_{L R}^{\sqrt{2}\left(k_{L}, k_{R}\right), \sqrt{2}\left(k_{L}^{\prime}, k_{R}^{\prime}\right)}\left(\mathcal{T}_{s}\right),
$$

where $N_{L R}^{\sqrt{2}\left(k_{L}, k_{R}\right), \sqrt{2}\left(k_{L}^{\prime}, k_{R}^{\prime}\right)}\left(\mathcal{T}_{s}\right)$ is defined analogously to $(6.5)$. Then eqs. $(6.6 b, c)$ will still hold when $\{\cdots\}_{h}$ is replaced with $\{\cdots\}_{s}$ (see eqs.(3.3) in [9]).

The point is the following. To any function of the form

$$
Z_{s}=\sum_{k_{L}, k_{R}, k_{L}^{\prime}, k_{R}^{\prime}} \alpha_{k_{L}, k_{R}, k_{L}^{\prime}, k_{R}^{\prime}}\left\{k_{L}, k_{R}, k_{L}^{\prime}, k_{R}^{\prime}\right\}_{s}
$$


we can assign the function

$$
Z_{h}=\sum_{k_{L}, k_{R}, k_{L}^{\prime}, k_{R}^{\prime}} \alpha_{k_{L}, k_{R}, k_{L}^{\prime}, k_{R}^{\prime}}\left\{k_{L}, k_{R}, k_{L}^{\prime}, k_{R}^{\prime}\right\}_{h}
$$

(The well-definedness of this assignment will be discussed below.) If $Z_{s}$ is modular invariant, then so will be $Z_{h}$.

Moreover, any theta-invariant $Z_{s} \in \Omega_{t h}\left(\mathcal{T}_{s}\right)$ and $Z_{h} \in \Omega_{t h}\left(\mathcal{T}_{h}\right)$ can be written in the forms of eqs. $(6.7 a, b)$, respectively. The proof of this is easy (see eq.(3.4b) in [9]), and is based on the fact that a subgroup of finite index in the modular group fixes each $\{\cdots\}_{h}$ and $\{\cdots\}_{s}$.

Thus eqs.(6.7) define a linear map from $\Omega_{t h}\left(\mathcal{T}_{s}\right)$ into $\Omega_{t h}\left(\mathcal{T}_{h}\right)$. This map is well-defined (hence onto) because, although $\{\cdots\}_{s}$ are not linearly independent, any choice of $\alpha$ for which the sum in $(6.7 a)$ is zero, will also give a zero sum in $(6.7 b)$. The reason for this is that the relations in (3.7d) of [9] hold in both the symmetric and heterotic cases, and generate all linear dependencies in the symmetric case. In general though there will be additional ones in the heterotic case, so this map will usually not be one-to-one.

Let $\Lambda_{s} \in \mathcal{L}_{e v}\left(\mathcal{T}_{s}\right)$, and define the lattice function $Z_{\Lambda_{s}}\left(\mathcal{T}_{s}\right)$ as in $(6.4 a)$. We proved in [9] that the theta-commutant of type $\mathcal{T}_{s}$ will be spanned by these lattice functions. For any such $\Lambda_{s}$, let $Z_{h}\left(\Lambda_{s}\right)$ denote the function in $(6.7 b)$ assigned to $Z_{\Lambda_{s}}\left(\mathcal{T}_{s}\right)$. Then these $Z_{h}\left(\Lambda_{s}\right)$ will necessarily span all of $\Omega_{t h}\left(\mathcal{T}_{h}\right)$.

Claim: For any $\Lambda_{s} \in \mathcal{L}_{e v}\left(\mathcal{T}_{s}\right)$, there exists a $\Lambda_{h} \in \mathcal{L}_{e v}\left(\mathcal{T}_{h}\right)$ such that

$$
Z_{h}\left(\Lambda_{s}\right)=L \cdot Z_{\Lambda_{h}}\left(\mathcal{T}_{h}\right)
$$

for some integer $L>0$.

Proof of claim Let $N_{h}$ be the coefficient matrix corresponding to $Z_{h}\left(\Lambda_{s}\right)$. Then a simple calculation gives us, $\forall \lambda_{L} \in \Lambda_{L}^{*} / \Lambda_{L}, \lambda_{R} \in \Lambda_{R}^{*} / \Lambda_{R}$,

$$
\left(N_{h}\right)_{\lambda_{L} \lambda_{R}}=\sum_{\lambda_{L}^{\prime}, \lambda_{R}^{\prime}}\left(N_{\Lambda_{0}}\right)_{\lambda_{L}^{\prime}, \lambda_{R}^{\prime}+2 \lambda_{R}}\left(N_{\Lambda_{s}}\right)_{\lambda_{L}, \lambda_{R}^{\prime} ; \lambda_{L}^{\prime},-\lambda_{R}}
$$

where the sum is over all $\lambda_{L}^{\prime} \in \Lambda_{L}^{*} / \Lambda_{L}, \lambda_{R}^{\prime} \in \Lambda_{R}^{*} / \Lambda_{R}$. But $N_{\Lambda_{0}}$ and $N_{\Lambda_{s}}$ look like $(6.4 b)$, so $(6.8)$ can be simplified to the rule:

$\left(N_{h}\right)_{\lambda_{L} \lambda_{R}}$ equals the number of $\lambda_{L}^{\prime}, \lambda_{R}^{\prime}$ for which both

$$
\begin{aligned}
\left(\lambda_{L}^{\prime} ; \lambda_{R}^{\prime}+2 \lambda_{R}\right) & \in \Lambda_{0}, \\
\left(\lambda_{L}, \lambda_{R}^{\prime} ; \lambda_{L}^{\prime},-\lambda_{R}\right) & \in \Lambda_{s} .
\end{aligned}
$$

Let $L=\left(N_{h}\right)_{00}$. Then $L \geq 1$ (since $\lambda_{L}^{\prime}=\lambda_{R}^{\prime}=0$ will always work for $\lambda_{L}=\lambda_{R}=0$ ). By linearity of (6.9), we see immediately that for each $\lambda_{L}, \lambda_{R}$, if $\left(N_{h}\right)_{\lambda_{L} \lambda_{R}}>0$, then $\left(N_{h}\right)_{\lambda_{L} \lambda_{R}}=L$. Therefore $N_{h} / L$ has only the entries 0 and 1 . 
Define $\Lambda_{h} \stackrel{\text { def }}{=}\left\{\left(\lambda_{L} ; \lambda_{R}\right) \mid\left(N_{h}\right)_{\lambda_{L} \lambda_{R}}=L\right\}$. Then $\left(\Lambda_{L} ; \Lambda_{R}\right) \subset \Lambda_{h}\left(\right.$ since $\left.\left(N_{h}\right)_{00}=L\right)$. We want to show that $\Lambda_{h}$ is a lattice. This will be so iff, for all $\left(\lambda_{L} ; \lambda_{R}\right),\left(\lambda_{L}^{\prime} ; \lambda_{R}^{\prime}\right) \in \Lambda_{h}$, both

$$
\begin{aligned}
\left(-\lambda_{L} ;-\lambda_{R}\right) & \in \Lambda_{h} \\
\left(\lambda_{L}+\lambda_{L}^{\prime} ; \lambda_{R}+\lambda_{R}^{\prime}\right) & \in \Lambda_{h}
\end{aligned}
$$

hold. But this is immediate from the linearity of eqs.(6.9).

Thus $\Lambda_{h}$ is a lattice, and $Z_{h}\left(\Lambda_{s}\right)=L \cdot Z_{\Lambda_{h}}\left(\mathcal{T}_{h}\right)$. Invariance under $\tau \rightarrow \tau+1$ immediately gives that $\Lambda_{h}$ contains only even norms, hence all its dot products are integers. Invariance under $\tau \rightarrow-1 / \tau$, and looking at the $\left(N_{\Lambda_{h}}\right)_{00}$ component, allows us to read off that $\left|\Lambda_{h}\right|=1$.

Therefore $\Lambda_{h}$ is even and self-dual. $\quad$ QED to claim

But any invariant in $\Omega_{W}\left(\mathcal{T}^{+}\right)$can be written as one in $\Omega_{t h}\left(\mathcal{T}_{h}\right)$, simply by multiplying it by $1=\chi_{g_{L 1}^{\prime}}^{0}$ etc. and using the Weyl-Kac formula to expand out the numerators of all characters into theta functions. Weyl-folding the claim then tells us that $\Omega_{e v}^{L}\left(\mathcal{T}_{h}\right)=$ $\Omega^{L}\left(\mathcal{T}_{h}\right)=\Omega_{W}\left(\mathcal{T}^{+}\right)$. Thm.D (ii) now completes the proof by saying $\Omega^{L}\left(\mathcal{T}^{+}+\mathcal{T}^{\prime}\right)=\Omega^{L}\left(\mathcal{T}_{h}\right)$. QED to Thm.E

\section{Comments}

In this paper we find a condition (4.3) which the algebras and levels must satisfy in order for heterotic physical invariants to exist. A lattice approach, called the generalized $R T W$ method, for finding heterotic physical invariants is proposed and analyzed. It will be summarized in the following paragraph. Using it, all heterotic physical invariants of total rank $n_{L}+n_{R}<4$ are found - see eqs.(5.3). We then prove in Thm.E that any heterotic physical invariant, of any type, can be obtained using this method.

The generalized RTW method: suppose we are interested in finding physical invariants of (positive) type $\mathcal{T}^{+}$. Then for any null type $\mathcal{T}^{\prime}$ for which the augment $\mathcal{T}^{+}+\mathcal{T}^{\prime}$ is accessible, find a self-dual gluing $\Lambda$ of the base lattice $\Lambda\left(\mathcal{T}^{+}+\mathcal{T}^{\prime}\right)$. Then find any $u$ satisfying (3.9) and compute the function $W Z_{\Lambda}^{u}\left(\mathcal{T}^{+}+\mathcal{T}^{\prime}\right)$, writing it as a linear combination of terms looking like (3.6). Each of these functions will be a modular invariant of type $\mathcal{T}^{+}$. Find the linear combinations of these invariants which satisfy properties (P2) and (P3). Any linear combination which does will be one of the desired physical invariants.

Examples of this method are provided in Sec.5. For small ranks and levels, this method is extremely practical [19]. There are other ways to find physical invariants (e.g. conformal embeddings), but a big advantage of this method is that it is complete: it will find all of them. But its greatest value may be theoretical, in that it offers a convenient description of the entire commutant. Indeed, a logical first step for classifying all physical invariants in a given class (see e.g. [4,20]) involves understanding the commutant, and lattices could provide a valuable tool for that. This is indicated in [21] by the transparency of a translation into the lattice language of the $A_{1}$ completeness proof, as well as in [9] by the classification given there of the level 1 symmetric physical invariants. 
It has been suggested in [21] that because the self-dual lattices involved here may be odd, this generalized RTW approach may be applicable to the study of coset theories. However, this question has not yet been adequately investigated.

An intriguing use for heterotic invariants of small rank has been suggested by C.S. Lam [22] as a means of reducing the rank of the effective gauge group for heterotic strings. The idea is to factorize the partition function of the theory: one factor will be a (small rank) physical invariant which describes the real world, while the other factor will be a physical invariant describing some 'shadow world'. These factors must be chosen so that the total central charge adds up to the correct numbers. For particle physics we can ignore the shadow world and its invariant - they would only be relevant for gravitational considerations such as the cosmological constant problem.

This work is supported in part by the Natural Sciences and Engineering Research Council of Canada. I would like to thank C.S. Lam for introducing me to the problem of heterotic invariants, and Patrick Roberts for many constructive criticisms. I have also benefited greatly from several conversations with Quang Ho-Kim. Finally, the hospitality of the Carleton mathematics department, where this paper was written, is much appreciated.

\section{Appendix}

A number of relevant quantities for each simple Lie algebra $g$ is collected below. By ' $\sim$ ' we mean the similarity relation discussed in Sec.4, and by $\left\{m_{1}, m_{2}, \ldots, m_{k}\right\}$ we mean the orthogonal lattice $I_{1}^{\left(m_{1}\right)} \oplus \cdots \oplus I_{1}^{\left(m_{k}\right)}$. ( $I_{1}$ is the 1-dimensional orthonormal lattice).

For $g=A_{n}(n \geq 1), \rho^{2}=n(n+1)(n+2) / 12, h^{\vee}=n+1,\left\|\Delta_{+}\right\|=n(n+1) / 2$, and $M=A_{n} . M \sim\{n+1, n+1, n+1\}$.

For $g=B_{n}(n \geq 3), \rho^{2}=n(2 n+1)(2 n-1) / 12, h^{\vee}=2 n-1,\left\|\Delta_{+}\right\|=n^{2}$, and $M=D_{n} . M \sim\{1\}$.

For $g=C_{n}(n \geq 2), \rho^{2}=n(2 n+1)(n+1) / 12, h^{\vee}=n+1,\left\|\Delta_{+}\right\|=n^{2}$, and $M=A_{1}^{n} \stackrel{\text { def }}{=} A_{1} \oplus \cdots \oplus A_{1}$ ( $n$ times). For $n$ even, $M \sim\{1\}$ and for $n$ odd $M \sim\{2\}$.

For $g=D_{n}(n \geq 4), \rho^{2}=n(n-1)(2 n-1) / 6, h^{\vee}=2(n-1),\left\|\Delta_{+}\right\|=n(n-1)$ and $M=D_{n} . M \sim\{1\}$.

For $g=E_{6}, \rho^{2}=78, h^{\vee}=12,\left\|\Delta_{+}\right\|=36$ and $M=E_{6} . M \sim\{3\}$.

For $g=E_{7}, \rho^{2}=399 / 2, h^{\vee}=18,\left\|\Delta_{+}\right\|=63$ and $M=E_{7} . M \sim\{2\}$.

For $g=E_{8}, \rho^{2}=620, h^{\vee}=30,\left\|\Delta_{+}\right\|=120$ and $M=E_{8} . M \sim\{1\}$.

For $g=F_{4}, \rho^{2}=39, h^{\vee}=9,\left\|\Delta_{+}\right\|=24$ and $M=D_{4} . M \sim\{1\}$.

For $g=G_{2}, \rho^{2}=14 / 3, h^{\vee}=4,\left\|\Delta_{+}\right\|=6$ and $M=A_{2} . M \sim\{3,3,3\}$.

The root lattices $A_{n}, D_{n}, E_{6}, E_{7}$ and $E_{8}$ are described at some length in [13].

\section{References}

[1] A.A. Belavin, A.M. Polyakov and A.B. Zamolodchikov, Nucl. Phys. B241 (1984) 333

[2] D. Gepner and E. Witten, Nucl. Phys. B278 (1986) 493 
[3] D.Gross, J.Harvey, E.Martinec and R.Rohm, Nucl. Phys. B256 (1985) 253;

D.Gross, J.Harvey, E.Martinec and R.Rohm, Nucl. Phys. B267 (1986) 75

[4] A. Cappelli, C. Itzykson and J.-B. Zuber, Nucl. Phys. B280 [FS18] (1987) 445;

A. Cappelli, C. Itzykson and J.-B. Zuber, Commun. Math. Phys. 113 (1987) 1

[5] P. Roberts and H. Terao, Int. J. Mod. Phys. A7 (1992) 2207;

N.P. Warner, Commun. Math. Phys. 130 (1990) 205

[6] A.N. Schellekens and S. Yankielowicz, Int. J. Mod. Phys. A5 (1990) 2903

[7] G. Moore and N. Seiberg, Nucl. Phys. B313 (1988) 16

[8] S. Bais and P. Bouwknegt, Nucl. Phys. B279 (1987) 561;

A.N. Schellekens and N.P. Warner, Phys. Rev. D34 (1986) 3092

[9] T. Gannon, WZW commutants, lattices, and level 1 partition functions (Carleton preprint, 1992)

[10] V.G. Kac, Infinite Dimensional Lie Algebras, 3rd ed. (Cambridge University Press, Cambridge, 1990)

[11] S.Kass, R.V. Moody, J. Patera and R. Slansky, Affine Lie Algebras, Weight Multiplicities, and Branching Rules Vol.1 (University of California Press, Berkeley, 1990)

[12] A.N. Schellekens, Classification of ten-dimensional heterotic strings (CERN preprint TH.6325, 1991)

[13] J.H. Conway and N.J.A. Sloane, Sphere packings, Lattices and Groups (SpringerVerlag, New York, 1988)

[14] T. Gannon and C.S. Lam, J. Math. Phys. 33 (1992) 871

[15] C.S. Lam, Strings constructed from free Bose and Fermi fields, Proceedings of Beijing String Workshop (World Scientific, 1987) (unpublished)

[16] D. Mumford, Tata Lectures on Theta Vol.1 (Birkhauser, Boston, 1984)

[17] J. Milnor and D. Husemoller, Symmetric Bilinear Forms (Springer-Verlag, Berlin, 1973)

[18] T. Gannon and C.S. Lam, Rev. Math. Phys. 3 (1991) 331

[19] Q. Ho-Kim and T. Gannon, The low level modular invariants of rank 2 algebras (work in progress)

[20] M. Bauer and C. Itzykson, Commun. Math. Phys. 127 (1990) 617

[21] P. Roberts, Whatever Goes Around Comes Around: Modular Invariance in String Theory and Conformal Field Theory, Ph.D. Thesis (Institute of Theoretical Physics, Goteborg, 1992)

[22] C.S. Lam (private communication) 